\title{
Selection and Designing of Command Shaper for Vibration Control of Flexible Manipulators: A Multi-objective Optimization Approach
}

\author{
Shafiul Alam \\ Department of Applied Physics, Electronics and Communication Engineering, The University of Dhaka, \\ Bangladesh
}

\author{
Osman Tokhi \\ Department of Automatic Control and Systems Engineering, The University of Sheffield, UK
}

(Received 28 September 2008; Accepted 22 June 2009)

\begin{abstract}
This paper presents a selection and design scheme of multimodal command shapers using the multi-objective genetic algorithm (MOGA). A control scheme comprised of a command shaper and a collocated proportionalderivative control is employed in order to reduce end-point vibration without sacrificing the system's response speed. Command shaping causes a delay in the system's response and, also, reduces system vibration and, in this manner, the amount of vibration reduction and the response delay conflict with each other. Conventional methods can hardly provide a solution that satisfies several design objectives demanded by practical applications due to the competing nature of these objectives. Furthermore, the selection of a shaping technique is crucial since robustness and computational complexity depend on the shaping technique. This paper proposes a combined approach to selecting and designing command shapers using MOGA. A comparative assessment of the performance of the proposed approach with the conventional single-objective and weighted-sum genetic algorithm optimization approaches is also provided. The proposed technique can provide a wide range of solutions in a single run to conflicting design objectives and satisfy associated goals.
\end{abstract}

\section{INTRODUCTION}

Flexible robotic manipulators are lighter, faster and less expensive than rigid ones but they pose various challenges in research as compared to rigid manipulators. In order to achieve high-speed and accurate positioning, it is necessary to control the manipulator's vibratory response in an effective manner. Input command shaping, introduced by Singer and Seering, ${ }^{1}$ has been applied to the control of different types of flexible systems for vibration reduction or trajectory tracking or, occasionally, both. ${ }^{2-4}$ The conventional command-shaping method involves convolving a desired command with a sequence of impulses, and the amplitudes and time locations of the impulses are calculated based on the natural frequencies and damping ratios of the system..$^{2-4}$ In this technique, the resonance poles of the system are cancelled regardless of the given reference input to the system. In flexible manipulator systems, the reduction in vibration at the end-point and the system's speed of response are generally found to conflict due to their construction. In such cases, conventional methods can hardly provide a solution that satisfies the several objectives and associated goals demanded by a practical application because of the competing nature of these objectives.

Evolutionary algorithms and swarm-intelligence optimization algorithms can be used to design command shapers as alternatives to conventional methods in order to minimise or maximise design objectives as demanded by practical applications. In the authors' previous work, assuming no prior infor- mation was available regarding the system, genetic algorithms (GA) were employed to derive amplitude and time locations of impulses, which were then convolved with the reference input to form the shaped signal..$^{5-7}$ In another work, a design method for command shaping was proposed in which multiple competing objectives were optimized simultaneously using the multi-objective genetic algorithm (MOGA) for a flexible manipulator system. ${ }^{8}$ The proposed method successfully yields a set of solutions that trade off among the competing objectives and satisfy design goals specified by the designer.

Suitable selection of a command shaper for any particular system/application is very important since robustness and computational complexity depend on it. Based on computational methods and performance specifications, there are three types of command shapers, 1) zero vibration (ZV), 2) zero vibration and derivative (ZVD) and, 3) extra-insensitive (EI). ${ }^{9}$ Although a large amount of work has been done on command shapers (both on design techniques and issues relating to practical applications) little work has been reported on the automated selection of the types of command shapers. This paper presents a combined approach to selecting and designing multi-modal command shapers applied to a flexible manipulator system. The aim of the control strategy is to reduce endpoint vibration with a satisfactory speed of response. MOGA is used as the main computational and decision-making unit of the proposed approach. A comparative study is provided with the conventional GA in the same application. The paper 